\title{
Review methods for image segmentation from computed tomography images
}

\begin{abstract}
Image segmentation is a challenging process in order to get the accuracy of segmentation, automation and robustness especially in medical images. There exist many segmentation methods that can be implemented to medical images but not all methods are suitable. For the medical purposes, the aims of image segmentation are to study the anatomical structure, identify the region of interest, measure tissue volume to measure growth of tumor and help in treatment planning prior to radiation therapy. In this paper, we present a review method for segmentation purposes using Computed Tomography (CT) images. CT images has their own characteristics that affect the ability to visualize anatomic structures and pathologic features such as blurring of the image and visual noise. The details about the methods, the goodness and the problem incurred in the methods will be defined and explained. It is necessary to know the suitable segmentation method in order to get accurate segmentation. This paper can be a guide to researcher to choose the suitable segmentation method especially in segmenting the images from CT scan.
\end{abstract}

Keyword: Computed tomography images; Image segmentation; Segmentation method 